\title{
Declining rates of gonorrhoea and syphilis in Strasbourg, France: a 20-year study
}

\author{
Bernard Cribier, Pierre-Henri Asch, Jean-Claude Tardieu
}

\begin{abstract}
Objective-To report the epidemiology of gonorrhoea and syphilis during the years 1973-1992 in the Strasbourg STD clinic and to discuss the data in the light of changes in STD rates in western countries.
\end{abstract}

Design-Retrospective analysis of gonorrhoea and syphilis cases recorded annually, total number of consultations, HIV serology, STD among prostitutes.

Setting-Strasbourg, Bas-Rhin, FRANCE. Subjects-Patients attending the public STD clinic in Strasbourg.

Results-From 1973 to 199279,786 consultations were recorded. Since 1984, a regular fall has been noted, from more than 4500 consultations $a$ year in the 1970s, to 1519 consultations in 1992. A major decline in gonorrhoea was observed, especially since 1984 . There were 457 new cases in $1973(15 \cdot 3 \%$ of all patients) whereas only seven new cases in $1992(0.8 \%$ of all patients). Syphilis has been declining regularly since 1979, with a reinforcement in this decline since 1984: 168 cases were recorded in 1972 (6.4\% of all patients), and only two cases in $1992(0.2 \%$ of all patients). Positive HIV tests were found in $14 \%$ and $12 \%$ of patients in 1985-86, and the positivity rate has regularly decreased to reach $0.6 \%$ in 1992. Prostitutes represented $9.2 \%$ of all patients in 1980 , and only $0.8 \%$ of patients in 1992. Since 1987, syphilis and gonorrhoea have been rarely recorded among prostitutes.

Conclusions-A dramatic decline in both gonorrhoea and syphilis has been observed in Strasbourg, especially since 1984, with a parallel diminution in the STD clinic frequentation. A comparable trend in gonorrhoea rates was noted in many western countries, but syphilis rates are more heterogeneous among geographic areas. Changes in sexual behaviour due to the AIDS epidemic are probably responsible in part for this decline, but nevertheless the rarity of gonorrhoea and syphilis in Strasbourg in 1992 is not clearly explained.

(Genitourin Med 1994;70:273-277)

\section{Introduction}

The epidemiology of sexually transmitted diseases (STD) is clearly related to many sociocultural factors and the incidence of gonorrhoea is known to change rapidly in response to various events. During the last decade, gonorrhoea rates were shown to decline in many European countries and in the USA. ${ }^{1}$ Data concerning syphilis are more contradictory: while syphilis is declining in Sweden, since 1987 the incidence of syphilis has increased in the USA. Major changes in STD epidemiology have been noted since the onset of the AIDS epidemic. A dramatic decrease in frequency of both syphilis and gonorrhoea was noted in Strasbourg (France) over the past eight years. In this article we present the epidemiological data of the STD clinic over the twenty year period between 1973 and 1992.

\section{Methods}

The STD centre of Strasbourg (Consultation des Maladies Infectieuses et Génitales) is a public clinic which provides free consultations to all patients suffering from STD in BasRhin, as in all French départments. The population of Bas-Rhin was 953,219 in 1992 $(881,634$ in 1975), and the clinic in Strasbourg is the only specialised centre for STD in the département. According to the recommendations of the medical administration (DDASS), epidemiological data were regularly provided to local health authorities. Complete data on the number of consultations, clinical findings, positive bacteriological investigations were therefore available for the last 20 years. Some sociocultural data were also analysed from the ten past years such as the professions of patients attending the clinic and particularly the number of prostitutes.

Patients mostly originated from the vicinity of Strasbourg. Data concerning medical demography were provided by CNAMTS (Caisse Nationale d'Assurance Maladie des Travailleurs Sociaux). The general population of Bas-Rhin was recorded by INSEE (Institute National de la Statistique et des Etudes Epidémiologiques). The diagnosis of gonorrhoea was established in all patients by bacteriological sampling and isolation of Neisseria gonorrhoeae, using VCF medium (Thayer and Martin). Syphilis was assessed by darkfield microscopy and serological testing: from 1973 to 1981 , Kline, Kolmer and Reiter reactions were used; since 1981 , both TPHA and VDRL replaced the older methods. HIV1 antibodies were detected with Abbott and Pasteur ELISA kits from 1985 to 1987 and since 1987, with a Fuji agglutination kit. A 


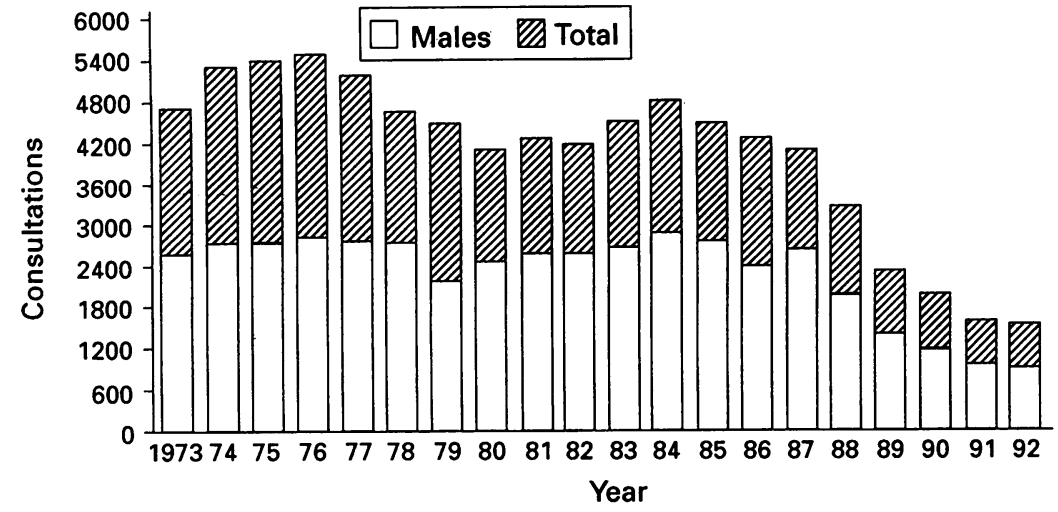

Figure 1 Overall number of annual consultations in the Strasbourg STD clinic, and number of consultations of male patients.

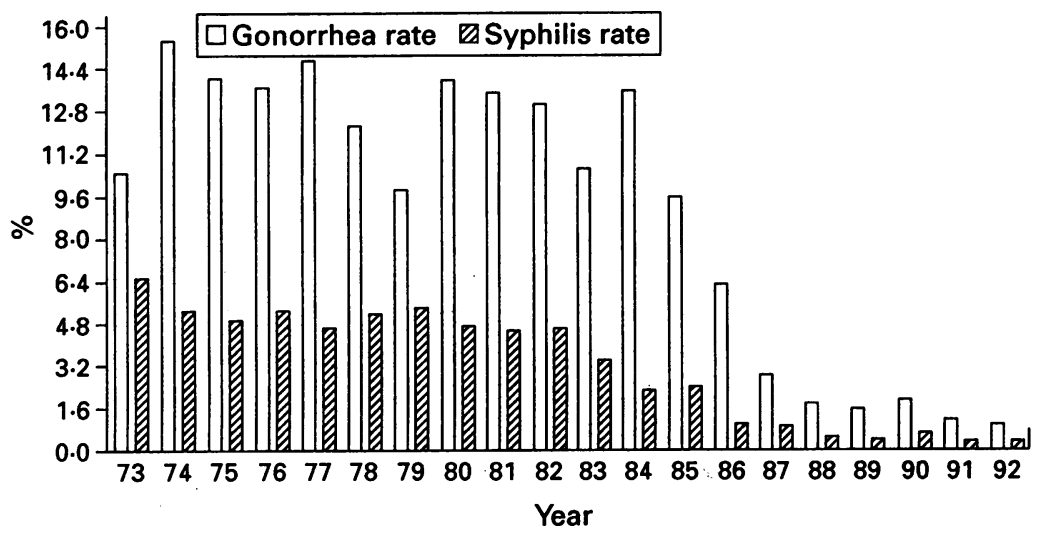

Figure 2 Syphilis and gonorrhoea rates among STD patients.

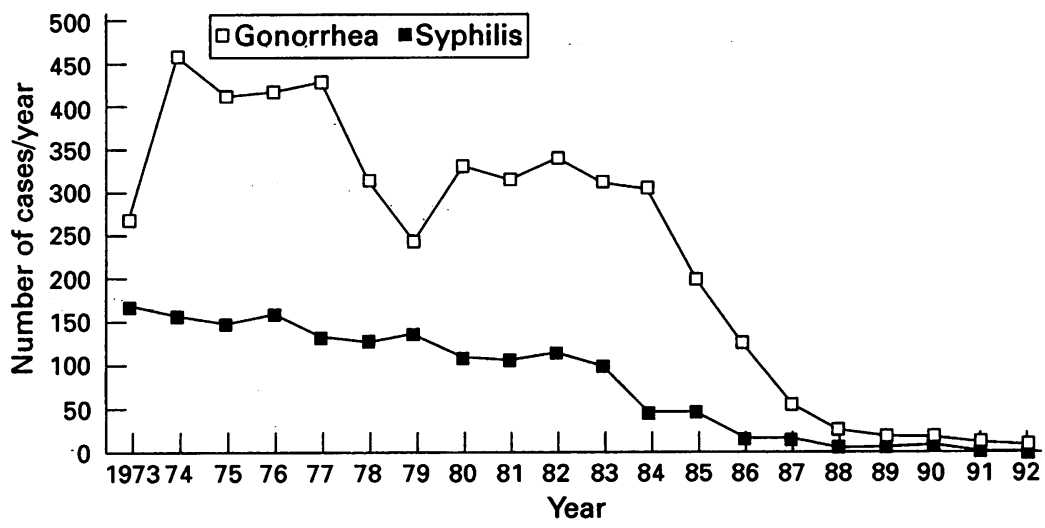

Figure 3 Total number of syphilis and gonorrhoea cases per year.

HIV1-HIV2 test was also developed since 1988 (Biotest).

\section{Results}

Total number of consultations per year (fig 1): 79,786 consultations were recorded from 1973 to 1992 . During the period 1973-1987, there were more than 4000 annual consulta- tions (range 4025-5367). Since 1984, the number of consultations has regularly decreased, especially in $1988(-21 \%), 1989$ $(-28 \%), 1990(-17 \%)$ and $1991(-19 \%)$. The average number of consultations per year for the period 1973-1977 was 5182, and only 2106 for the period $1988-1992(-59 \%)$. The male/female ratio was 1.4: 1 for the 20-yearperiod (males $=57.5 \%$ ), but a slight increase has been observed since the 1980 s (fig 1). The average number of consultations per year per patient ranged from 1.6 to $2 \cdot 1$ (average = 1.78 ), without significant change during the reference period.

Gonorrhoea (fig 2). The number of gonorrhoea cases fell from more than 400 annual cases in the 1970s to seven in the year 1992. The onset of the decrease was the year 1985 $(-34 \%)$, and dramatic falls were observed in $1987(-61 \%)$ and $1988(-48 \%)$. A slight decrease had already been noted in 1978 , but the number of gonorrhoea cases remained stable from 1979 to 1984 . The sex ratio showed a male predominance (approximately 2:1), which remained unchanged during the 20 years. The rate of gonorrhoea in patients consulting the STD clinic was at its maximum in $1974(15.3 \%)$ and has been falling regularly since 1984 to reach $0.8 \%$ in 1992 (fig 2).

Syphilis (fig 2). Since 1979 the incidence of syphilis has decreased in Strasbourg: 168 cases were observed in 1973, and only 2 in 1992 and a dramatic fall in the total number of cases has been noted since 1984. Syphilis can be considered as a rare disease since 1988 , with only three cases of primary syphilis observed in a 5 year period. In comparison, 15 to 30 cases of primary syphilis were annually recorded during the 1970 s. The distribution of cases between the various stages of syphilis has remained stable during the 20 years (table 1): most of the cases were latent syphilis, with tertiary cases being rarely observed. The sex ratio remained unchanged from 1973 to the 1990 s and was approximately 2:1. When considering the rate at which syphilis was diagnosed in patients consulting the STD clinic, this fell from $6.4 \%$ in 1973 to $0.2 \%$ in 1992 , with an increase in this decline since 1982 (fig 3 ).

HIV tests (table 2). Commercial kits for the detection of HIV antibodies have been available since May 1985. The rate of HIV positivity was high in 1985 and 1986 (14\% and $12 \%)$, when all patients with high risk factors were tested. Since 1989, only a few HIV positive patients have been detected. The overall number of HIV tests has decreased since 1988 , because free and anonymous tests have been provided in Strasbourg since 1988 by

Table 1 Distribution of patients according to syphilis stages and associated sex ratios (\% of male cases).

\begin{tabular}{|c|c|c|c|c|c|c|c|c|c|c|c|c|c|c|c|c|c|c|c|c|}
\hline & 1973 & 1974 & 1975 & 1976 & 1977 & 1978 & 1979 & 1980 & 1981 & 1982 & 1983 & 1984 & 1985 & 1986 & 1987 & 1988 & 1989 & 1990 & 1991 & 1992 \\
\hline I & 15 & 29 & 41 & 20 & 15 & 15 & 18 & 15 & 9 & 7 & 9 & 11 & 6 & 2 & 2 & 1 & - & 1 & 1 & - \\
\hline II & 34 & 29 & 35 & 43 & 50 & 28 & 47 & 26 & 17 & 19 & 20 & 15 & 10 & 2 & 6 & - & 1 & 1 & - & - \\
\hline III & 5 & 3 & 2 & 3 & 2 & 1 & $\frac{\pi}{\pi}$ & 5 & $\frac{11}{n}$ & 2 & 2 & 1 & - & - & - & 2 & - & 1 & - & - \\
\hline latent & 109 & 93 & 70 & 81 & 63 & 86 & 70 & 53 & 80 & 87 & 68 & 21 & 32 & 14 & 9 & 3 & 4 & 3 & 1 & 2 \\
\hline Congenital & 5 & 1 & 3 & 2 & 2 & - & - & - & - & - & - & - & - & - & - & - & - & - & - & - \\
\hline $\begin{array}{l}\text { Sex ratio } \\
\text { (\% males) }\end{array}$ & 62 & 68 & 74 & 61 & 65 & 82 & 73 & 72 & 73 & 76 & 64 & 65 & 73 & 56 & 77 & 83 & 60 & 0 & 50 & 50 \\
\hline Total & 168 & 155 & 148 & 159 & 132 & 129 & 135 & 109 & 106 & 115 & 99 & 48 & 48 & 18 & 17 & 6 & 5 & 6 & 2 & 2 \\
\hline
\end{tabular}




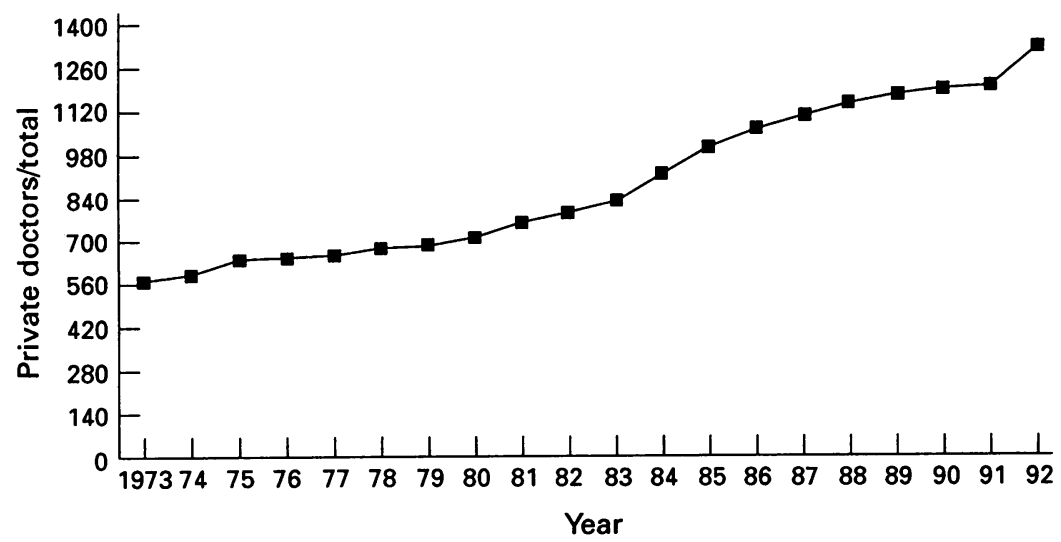

Figure 4 Total number of private doctors in Bas-Rhin (general practitioners + dermatologists + gynaecologists + urologists).

Table 2 HIV prevalence in the Strasbourg STD clinic.

\begin{tabular}{llllllllc}
\hline HIV Prevalence & May-Dec 85 & 86 & 87 & 88 & 89 & 90 & 91 & 92 \\
\hline Tests (n) & 241 & 260 & 425 & 495 & 255 & 159 & 95 & 158 \\
HIV-Positive (n) & 34 & 31 & 15 & 10 & 2 & 1 & 4 & 1 \\
HIV-positive (\%) & 14 & 12 & 3,5 & 2 & 0,7 & 0,6 & 4 & 0,6 \\
\hline
\end{tabular}

the Dermatology department. These data have not been combined with the STD clinic recordings, since they only concern HIV testing in STD-free patients.

Prostitution (table 3). Data on prostitution have been regularly available since 1980 . In $1980,9.2 \%$ of the patients from the STD clinic were prostitutes, whereas in 1993, only eight were prostitutes $(0.8 \%$ of all patients). The gonorrhoea rate among prostitutes was high in 1980 (15\%), whereas syphilis was already rarely noted. Since 1987 , syphilis and gonorrhoea had been exceptionally isolated in prostitutes.

\section{Discussion}

National French epidemiological data on STD (except AIDS) certainly underestimate the incidence, since most cases are no longer notified to the Health Care authorities. The only relevant data originate from STD centres or laboratories. From 1986 to 1990 , a group of more than 300 French STD clinics recorded a two-fold decrease of their activity ${ }^{2}$ with a parallel decline in the number of STD diagnosed. The decrease in the total number of consultations in Strasbourg is similar during the same period, whereas it had remained stable during the 15 previous years.

Gonorrhoea Since 1985, a national network of laboratories has been established for the surveillance of gonorrhoea in France ("RENAGO"). The epidemiological data obtained by RENAGO showed a decrease in the number of isolations of gonococci from 14.9 cases per year per laboratory in 1986 to 2.7 in $1990 .{ }^{3}$ The estimated incidence of gon- orrhoea reported by private general practioners decreased during the same period from 0.48 to 0.12 cases per month per practioner. ${ }^{2}$ A similar decrease was noted in many European countries: in Sweden (1), the United Kingdom, ${ }^{45}$ Belgium and Switzerland, ${ }^{6}$ the Netherlands ${ }^{7}$ and in the United States, ${ }^{1}$ Israel ${ }^{8}$ and Australia. ${ }^{9} 10$ This diminution was first noticed in the mid 1970s, thus preceding the AIDS epidemic. A major and rapid decline has been observed since the mid 1980s, which is probably related to a change in sexual behaviour due to the AIDS risk. An extraordinary 15-fold decline was noted in Sweden (487 cases per 100,000 in 1970 to 31 cases per 100,000 in 1987), with a parallel decrease in the male:female ratio. ${ }^{1}$ Epidemiological data from the main STD clinics in the Netherlands revealed a decline of $78 \%$ in gonorrhoea incidence in the period 1981-1987, with a constant sex ratio. ${ }^{7}$ The decline was weaker in the United States and in Canada, with an approximate 2-fold decrease in the incidence of gonorrhoea during the past decade. ${ }^{1}$ An overall diminution of $90 \%$ in case numbers was recorded in Sydney, Australia. ${ }^{9}$ Even in Asia, gonorrhoea is decreasing. Cases reported in Singapore can be considered good epidemiological markers and the incidence has fallen from 684 cases per 100,000 in 1979 to 318 in $1985 .^{1}$

In the Strasbourg STD clinic, an extraordinary decrease of gonorrhoea was observed, and this was much more significant than the decrease noted in other European cities such as Amsterdam ${ }^{7}$ and London. ${ }^{4}$ In Strasbourg the number of new cases in 1992 was 65 -fold lower than in 1974, and the rate of gonorrhoea in STD patients was 19-fold lower. The gonorrhoea rate seemed to reach a peak in the period 1974-1977 and a continuous decline has been noted since 1978, which has been accelerated since 1984. As in many Western countries, the decrease in gonorrhoea preceeded the onset of the AIDS epidemic. The major decline since 1984 is probably related to the large media news coverage on AIDS in the period 1984-1986 when AIDS became a public health problem. At the same time, attendance at the STD clinic decreased, while the population of Bas-Rhin increased by $8 \cdot 1 \%$ between 1975 and 1990. The decrease in clinic attendance was especially marked for prostitutes and other people with high STD risk factors, such as multipartner homosexuals. Since the total number of consultations between 1984 and 1992 decreased in a parallel fashion for men and women, these results probably reflect changes in sexual behaviour of both homosexual and heterosexual patients. Homosexual males were probably aware of AIDS and its sexual transmission

Table 3 Gonorrhoea and syphilis cases among prostitutes.

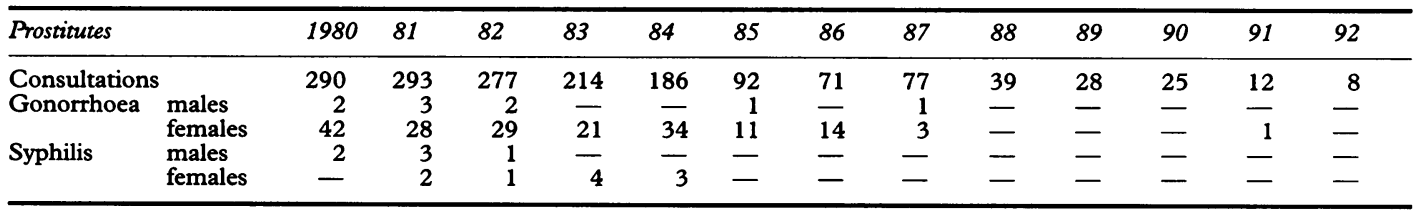


before other people, and changed their sexual behaviour sooner, as has been demonstrated in large US cities. ${ }^{1}$ This hypothesis is supported by the decline in the HIV-positivity rate in Strasbourg STD clinic patients in 1988 and a similar trend was observed in London between 1982 and $1987 .{ }^{4}$ The sex ratio of gonorrhoea remained stable in Strasbourg, with a male predominance as observed in Amsterdam. ${ }^{6}$ The hypothesis of an artificial decrease in gonorrhoea has been proposed, because educational programmes and epidemiological recordings are concentrated principally on AIDS and syphilis. ${ }^{11}$ However, this explanation is not satisfactory since urethral samples are systematically taken in most STD clinics including Strasbourg. When people with gonorrhoea were carefully questioned, ${ }^{12}$ complex behaviours and sexual contexts were noted. Intravenous drug use, casual sex partners, condom use, sexual activity after onset of symptoms or knowledge of STD exposure may influence the transmission of the disease. ${ }^{12}$ It is difficult to know the exact part each factor plays in the changes in gonorrhoea incidence. In Strasbourg, data about sexual behaviour of STD patients are not available and therefore the fall in gonorrhoea incidence remains partially unexplained.

The decline of gonorrhoea could reverse in the next years, since penicillinase-producing Neisseria gonorrhoeae (PPNG) strains are increasing in most countries, ${ }^{13}$ and asymptomatic cases are more common than in past reports. ${ }^{14}$ PPNG strains are also increasing in France, despite the fall in the total number of cases of gonorrhoea, ${ }^{3}$ especially among patients from the major STD clinics in Paris.

When considering the rate of gonorrhoea among all STD, major changes have been observed in Strasbourg. Data on chlamydial and papillomavirus infections are not comparable for the 20-year period, since the methods used for diagnosis have considerably changed. In the Strasbourg STD clinic, the ratio of chlamydial/gonococcal infections has increased from 1 in 1984 to 8 in 1990 in men and from 2 to 7 in women, ${ }^{15}$ while the total number of STD decreased.

Syphilis.

Data on syphilis are more heterogeneous among different countries. After the general fall in incidence due to the introduction of penicillin, the incidence began again to increase in many Western countries during the early 1960s to the end of the 1970s. In Sweden, syphilis incidence fell again in 1982 sharply after the recognition of the AIDS epidemic, ${ }^{1}$ while in the USA the incidence remained stable from 1965 to 1977. An increase of primary and secondary cases was noted in the USA from 1978 to 1982, followed by a decline, especially in men since 1982 , probably as a result of changes in the sexual behaviours of homosexual and bisexual men. ${ }^{11}$ However, in contrast to the decline in gonorrhoea, a marked increase in syphilis incidence was noted in 1987 in the USA, espe- cially in black men and women. ${ }^{1}$ This was confirmed in 1988, 1989 and $1990 .{ }^{116}$ Racial differences in syphilis incidence increased during the period $1981-1989$ in the USA, ${ }^{11} 16$ with major regional differences. In four states the incidence among black men was more than 400 per 100,000 whereas syphilis is a rare disease in most other states with incidences of less than 2 cases per 100,000. The increasing rates of syphilis are probably linked to the use of illegal drugs, particularly the "crack" cocaine, and to limited access to health care. ${ }^{16}$ In the United Kingdom, the syphilis rate remained stable during the period 1976-1982 and has begun to decrease since 1982 , with a 2 -fold decline in $1986 .{ }^{1}$ When considering the VDRL-positive rates in blood-donors in South Korea, a marked decrease was noted during the period 1977-1990. ${ }^{17}$ In France, a 3-fold decrease of primary syphilis was noted in the STD clinics in Paris between 1985 and $1987 . .^{18}$ The rate of primary syphilis remained stable in the French STD clinics during the period 1986-1990 (10 cases per 1000 consultations), while total syphilis cases showed a slight fall from $26 / 1000$ to $19 / 1000 .^{2}$ Changes in syphilis incidence are related to changes in sexual behaviour ${ }^{19}$ and to various socio-economic, racial or geographic factors, yet the racial difference in the USA is poorly understood.

Syphilis was already declining in the 1970s in Strasbourg, before the decline in gonorrhoea and the AIDS epidemic. The fall in total cases per year has been continuous since 1979, with a major reinforcement in this decline in $1984(-51 \%)$. The rate of syphilis also dramatically declined from 36 cases per 1000 consultations in 1973 to 1 per 1000 in 1992. There is no satisfactory explanation for this trend, since no particular educational programme was undertaken in Strasbourg during these years. The decline in the $1980 \mathrm{~s}$ is probably related to general changes in sexual behaviour since people became aware of AIDS. Nevertheless, the near disappearance of syphilis (total number of cases was 84 fold lower in 1992 than in 1973, and the rate of syphilis in STD patients was 32 fold lower) is not understood. Cultural factors are probably partly responsible for the changes observed in Strasbourg since people are known to be more "disciplined" in Alsace. Moreover, the HIVpositivity rate is lower in Alsace than in other parts of France such as Paris and the south of France. In 1993, the rate of cumulated cases of AIDS was 141 per million in the département of Bas-Rhin, 1438 per million in the Alpes Maritimes and 2921 per million in Paris. ${ }^{20}$ The prevalence of intravenous drug use is also lower in Strasbourg than in other large French cities.

\section{Prostitution.}

The reduction in STD rates in prostitutes and the parallel decline in their frequentation of the STD clinic probably reflect the use of condoms among sex workers in Strasbourg. There have been no legal modifications con- 
cerning the control of prostitution in the past 13 years in France which might otherwise explain this change of behaviour.

\section{An artefactual decline?}

A possible explanation for an artefactual decline in both the number of STD consultations and the gonorrhoea and syphilis rates could be the increasing number of private practioners in Strasbourg and in the BasRhin. Analysis of the medical demography of the Bas-Rhin during the period 1973-1992 has shown an marked increase in the number of general practitioners (502 to 1129), gynaecologists (37 to 124) and dermatologists (22 to 61). Nevertheless, this two- to three fold increase in the number of private practioners has been continuous during this period, while the decrease in gonorrhoea and syphilis incidence were strongly reinforced after 1984 . The decline was also much more pronounced than a simple distribution of STD cases among new practitioners. Moreover, the patients of the STD clinic are mainly represented by people of lower socio-economic status who care about free consultations and treatments. The preferential orientation of the STD patients toward private practitioners since 1984 seems therefore very improbable and French national data have also showed a decrease in gonorrhoea reported by private doctors. $^{2}$ During this 20-year period, the recording of STD cases was carefully done, and underdiagnosis of gonorrhoea and syphilis is unlikely since the methods used for diagnosis in the 1980 s were more sensitive than in previous years. Although the decrease in gonorrhoea and syphilis incidences were more significant than those reported by other French STD clinics, these decreases recorded in Strasbourg are probably not artefactual.

\section{Conclusion}

The data presented reflect major changes in STD incidence in the eastern part of France. These observations were made principally in young people of lower socioeconomic classes, especially students, unemployed and prostitutes who represent about $50 \%$ of the patients in the STD clinic. A reduction in the incidence of STD was noted, as was a reduction in the relative frequencies of syphilis and gonorrhoea among STD, and a decrease in the frequency of STD in prostitutes. These declines are corroborated by decreases in the overall number of STD cases in all French
STD clinics since 1985 . While changes in sexual behaviours could be more significant in Alsace because of cultural reasons, this exceptionally dramatic decline in two major STD remains partially unexplained.

We are grateful to Dr Pignol for providing information on medical demography. We thank Mrs Marie-Odile Adam, Mrs Christine Claverie and Mrs Eliane Tagland very much for their patience and help in collecting the patient data. We thank their patience and help in collecting the patient data. We thank Mrs Nathalie Najberg for excellent assistance in the prepara-
tion of the manuscript and Dr J. Byrne for critically reading tion of the man
the manuscript.

1 Aral SO, Holmes KK. Epidemiology of sexual behavior and sexually transmitted diseases. In Holmes KK, Transmitted Diseases. New York. Mac Graw Hill 1990, 2nd ed.

2 Marshall B, Lepoutre A. Dispensaires antivénériens. Evolution de leur activité et de l'incidence de certaines maladies sexuellement transmissibles en France (1986-1990). Bulletin Epidémiologue Hebdomadaire 1992, nr 9: 39-41.

3 Goulet V, Mamet JP, Rebiere I, Sednaoui P, Catalan F. Le gonocoque en France en 1990: données du réseau RENAGO. Bulletin Epidémiologue Hebdomadaire 1992, nr 17:73-4

4 Carne CA, Johnson AM, Pearce F, et al. Prevalence of antibodies to HIV, gonorrhoea rates, and changed sexual behavior in homosexual men in London. Lancet 1987; i:656-8.

5 Sexually transmitted diseases in Britain: 1985-86 Genitourin Med 1989;65:117-21.

6 Walckiers D, Piot P, Stroobant A, Van der Veken J, Declercq E. Declining trends in some sexually transmitted diseases in Belgium between 1983 and 1989 Genitourin Med 1991;67:374-7.

7 Van de Laar MJW, Pickering J, Van de Hoeck JAR, Van Griesven GJP, Coutinho RA, Van de Water HPA Declining gonorrhoea rates in The Netherlands, 1976-88: consequences for the AIDS epidemic. Genitourin Med 1990;66:148-55.

8 Green MS, Karsenty E, Slater PE. Recent decline in gonorrhoea incidence in Israel: possible association with the AIDS pandemic. Isr $\mathcal{F} M e d$ Sci 1988;24:137-140.

9 Donovan B, Beck MD, Pethebridge AM, Nelson MJ. Heterosexual gonorrhoea in central Sydney: implication feterosexual gonorrhoea in central Sydney: imp

10 Donovan B, Harcourt C, Basset I, Philpot CR Gonorrhoea and asian prostitution: the Sydney health center experience. Med $\mathcal{F}$ Austr 1991;154:520-1.

11 Rolfs RT, Nakashima AK. Epidemiology of primary and secondary syphilis through the United States, 1981 through 1989. ЭAMA 1990;264:1432-7.

12 Upchurch DM, Brady WE, Reichart CA, Hook EW III Behavioral contributions to acquisition of gonorrhoea in patients attending an inner city sexually transmitted disease clinic. $\mathcal{F}$ Infect Dis 1990;161:938-41.

13 Handsfield HH, Rice RJ, Robertys MC, Holmes KK Localized outbreak for introduction of Penicillinase-producing Neisseria gonorrhoea. $¥ A M A$ 1989;261:2357-61.

14 Sherrard J, Barlow D. Gonorrhoea in man. Lancet 1993;341:245.

15 Le Faou AE, Bottlaender M, Tardieu JC. Incidence of Neisseria gonorrhoeae and Chlamydia trachomatis infections in Strasbourg over 8 years. Genitourin $\mathrm{Med}$ 1991;67:428-30.

16 Hook EW III, Mara CM. Acquired syphilis in adults. $N$ Engl f Med 1992;326:1060-9.

17 Lee JB, Byeon SW, Chung KY, Lee MG, Whang KK Syphilis prevalence is rapidly decreasing in South Korea. Genitourin Med 1992;68:60.

$18 \mathrm{X}$. Tendances de l'incidence de certaines maladies sexuellement trasmissibles (MST) en France. Bulletin Epidémiologue Hebdomadaire 1989, $\mathrm{nr}$ 3:9-10.

19 Mindel A, Tovey SJ, Williams P. Primary and secondary syphilis, 20 years' experience. 1. Epidemiology. Genitourin Med 1987;63:361-4.

$20 \mathrm{X}$. Surveillance du SIDA en France (Situation au 31 mars 1993). Bulletin Epidémiologue Hebdomadaire 1993, nr 18:77-83. 